\title{
Cuídate, hacete un PAP: un abordaje colectivo para pensar y repensar la problemática del cáncer de cuello de útero
}

María Fernanda Abrigo Robles

Docente de la carrera de Trabajo Social de la Facultad de Ciencias Jurídicas y Sociales. Universidad Nacional del Litoral.

Trabajadora Social en la Dirección de las Residencias de Salud del Ministerio de Salud de la Provincia de Santa Fe.

Gabriel Jeanney

Docente de la Facultad

de Ciencias Médicas.

Universidad Nacional del Litoral.

Médico del Laboratorio de

Procesamiento de Papanicolaou

Dr. Daniel Rocco dependiente del

Ministerio de Salud de la Provincia

de Santa Fe.

\author{
Delia María Passeggi \\ Docente de la carrera de Trabajo \\ Social de la Facultad de Ciencias \\ Jurídicas y Sociales. Universidad \\ Nacional del Litoral. \\ Trabajadora Social de "Casa del Sol", \\ Centro de Tratamiento Ambulatorio \\ de Adicciones del Ministerio de Salud \\ de la Provincia de Santa Fe.

\section{Florencia Bustos} \\ Licenciada en Obstetricia del \\ Centro de Salud Callejón Roca \\ y del Hospital Iturraspe.
}

Universidad y salud /

Intervenciones

\section{Resumen}

El presente artículo deriva de la experiencia de un proyecto de extensión de la Universidad Nacional del Litoral denominado Prevención de cáncer de cuello uterino: estrechando lazos entre el equipo de salud y las mujeres. Experiencia de trabajo en el Centro de Salud San Agustín. El proyecto se viene realizando desde el año 2013 y lo llevan adelante docentes, profesionales y estudiantes de la carrera de Trabajo Social y la Facultad de Ciencias Médicas.

El interés del mismo surge a partir de identificar al cáncer de cuello de útero como aquella problemática que refleja la desigualdad social en salud. A nivel mundial es la segunda causa de muerte por cáncer, a pesar de que la enfermedad podría prevenirse casi por completo gracias a las políticas públicas, conocimientos y tecnologías actualmente disponibles.

Este proyecto se orienta hacia aquellas mujeres de 35 a 64 años pertenecientes a los barrios San Agustín I y San Agustín II. Este grupo etario es el de mayor riesgo y menor tránsito por el espacio preventivo de cáncer de cuello uterino. La complejidad de la problemática muestra la necesidad de desarrollar acciones que ponga el foco en la prevención y atención del cáncer de cuello de útero. Acción que requiere de un trabajo en red, interinstitucional e interdisciplinario sostenido en el tiempo.

\author{
Palabras clave \\ - mujer \\ - identidad de género \\ - salud \\ - cáncer de cuello uterino \\ - prevención
}

\section{Introducción}

El presente artículo da cuenta de la experiencia en un Proyecto de Extensión de Interés Social (PEIS) denominado Prevención de cáncer de cuello uterino: estrechando lazos entre el equipo de salud y las mujeres. Experiencia de trabajo en el Centro de Salud San Agustín, proyecto que tiene relación con dos Programas de la Secretaría de Extensión de la Universidad Nacional del Litoral (UNL): Equidad en Salud y Género, universidad y sociedad. El interés del mismo surge a partir de identificar al cáncer de cuello de útero como aquella problemática que refleja con máxima crudeza la desigualdad social en salud.

Se ha detectado que afecta principalmente a mujeres en situación de pobreza, socialmente vulnerables, que no acceden a los servicios de tamizaje. ${ }^{1}$ La enfermedad y muerte de estas mujeres tiene consecuencias devastadoras para las condiciones de vida de sus familias, ocasionando la pérdida de trabajo, ingresos y el ausentismo escolar de los niños del hogar (Arrossi y otros, 2007).

1) Se entiende por pruebas de tamizaje aquellos exámenes aplicados con el fin de identificar una población, aparentemente sana, en mayor riesgo de tener una determinada enfermedad, que hasta ese momento no se les ha diagnosticado.
En este sentido, el cáncer de cuello de útero no sólo afecta a las mujeres más pobres, sino que es a su vez un factor que intensifica la pobreza y la vulnerabilidad social.

Argentina no es una excepción a esta realidad. Cada año se diagnostican alrededor de 3000 casos nuevos de cáncer de cuello de útero (Ferlay y otros, 2004), y alrededor de 1600 mujeres mueren a causa de la enfermedad. En los últimos treinta años no ha habido descensos significativos de la mortalidad por esta causa. Sin embargo, a pesar de la falta de impacto de las acciones preventivas organizadas en nuestro país y en las provincias durante las últimas décadas, hoy se intenta comprender el porqué de dicho fracaso, a partir de un Programa Nacional de Prevención que sustenta su planificación y desarrollo en la corrección de yerros pasados. Equívocos que tuvieron su causa a partir de la falta de estudios sobre una problemática que es mucho más compleja de lo que en un principio se pensó. No hubo antes un análisis sistemático para 


\section{6}

el interés del proyecto surge a partir de identificar al cáncer de cuello de útero como aquella problemática que refleja con máxima crudeza la desigualdad social en salud

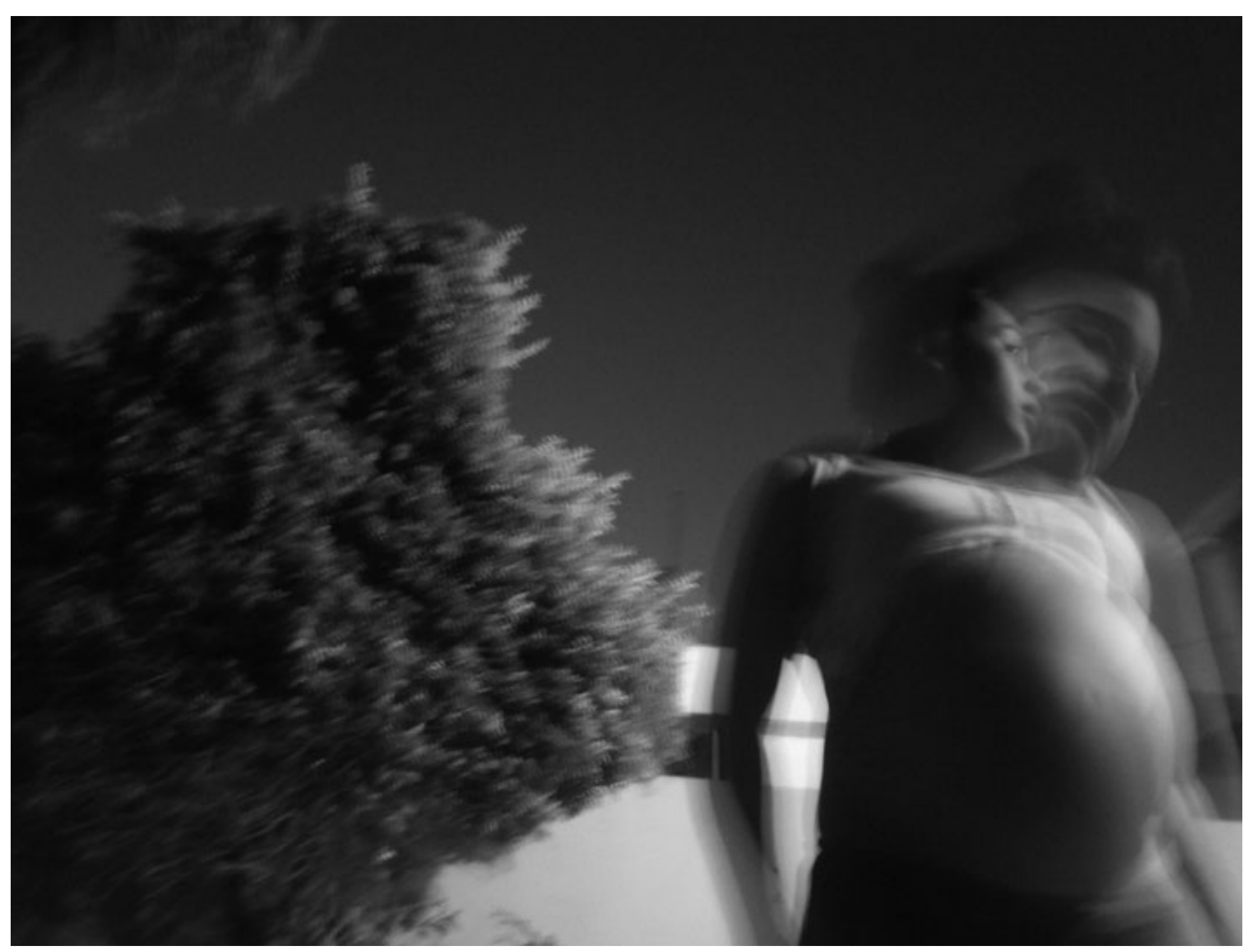


comprender en profundidad las razones de este fracaso. Hoy se está pensando y trabajando en función de aquello, a partir de la unificación de criterios en el diagnóstico de situación, planificación de metodologías de tamizaje y desarrollo de laboratorios centralizados de citodiagnóstico en patología cérvico vaginal con sistemas de contralor y estadísticas unificadas que incluyen la historia y seguimiento de la salud de nuestras mujeres.

En principio, hay que entender que la mujer es un sujeto de derecho y necesidad. Que no es solamente importante durante su embarazo o la lactancia, relacionada como binomio "madre e hijo" o ser la depositaria de la salud de la familia, entendiendo y comprendiendo que muchas son jefas de hogar y aunque no lo sean, trabajan como si lo fueran. La morbimortalidad por cáncer de cuello de útero es un claro ejemplo de una patología previsible, prevenible y curable, que muestra no sólo las debilidades de un sistema de salud con referencia a las respuestas que debe brindarle a ese sector de la población, sino que además, pone de manifiesto las desigualdades y las inequidades que posicionan a la mujer en un mundo que la determina y condiciona en una búsqueda constante y que constituye uno de los principales determinantes de su salud. Pero desconocer las debilidades de este sistema de salud sería no saber cómo intervenir o, sería intervenir sin comprender las implicancias que pudiesen comprometer el funcionamiento de un centro de salud determinado y su bidireccionalidad con la comunidad. Conocer al centro de salud y a sus integrantes es de fundamental importancia ya que ningún equipo es igual a otro, en cuanto a la transferencia que existe entre sus integrantes y la representación y significación que los vincula estrechamente a la Atención Primaria de la Salud. De la misma manera, la comunidad que rodea todo centro, muestra su singularidad y ésta debe ser descubierta y comprendida. Aceptar las debilidades del sistema implica además entender las limitaciones en recursos humanos, estructuras e insumos. Es aceptar que cuando se planifica una estrategia de prevención con relación a una determinada patología se debe entender que ésta debe ser inserta en la dinámica de trabajo sin afectar el abanico funcional, en la diversidad de la salud que debe ser atendida.

Se asume a la salud como un derecho, como un bien social, como "la capacidad de luchar contra las condiciones que limitan la vida" (Rovere, 2006); y por lo tanto, desde esta concepción compleja del proceso salud-enfermedad-atención, es que no se puede pensar a la mujer escindida de su contexto histórico, político, económico, ideológico, cultural.

Según datos de la Dirección General de Estadísticas del Ministerio de Salud, en la provincia de Santa Fe, la tasa de mortalidad

2) ASCUS: Células escamosas de significado indeterminado.

3) L-SIL: Lesión escamosa intraepitelial de bajo grado. Se consideran anormalidades leves causadas por infección de VPH.

4) Lesiones preneoplásicas: alteracio- nes que se producen en algún tejido que alteran su normalidad haciendo más probable que sobre ellas se desarrolle alguna neoplasia o cáncer. 5) No se pudo precisar cantidad de población debido a que las fuentes consultadas no disponen de datos

estandarizadas por cien mil mujeres es de 8,2 para el año 2010; y en general el grupo etario más afectado es el de 45 a 49 y 55 a 59 años, con una tasa de mortalidad especifica de 11,3 y 13 por cien mil mujeres respectivamente; con un promedio provincial de cobertura con Papanicolaou (PAP) cercano al $40 \%$; un porcentaje muy bajo de mujeres embarazadas cubierta con esta práctica del 13 \% para el año 2011, en el Nodo Santa Fe, siempre referidas a la población con cobertura pública o sin obra social.

\section{Recorte geográfico y etario del proyecto de extensión: una mirada al interior del Centro de Salud San Agustín} El Centro de Salud San Agustín se encuentra ubicado en el barrio del mismo nombre, en el distrito noroeste de la ciudad de Santa Fe. Para este proyecto se tomó como base los datos elaborada por el Laboratorio de Papanicolaou Dr. Daniel Rocco del Ministerio de Salud de la Provincia en el período 2009-2012. Como puede apreciarse en la tabla 1, durante este período la producción de PAP fue muy baja.

El Centro pertenece a uno de los barrios más vulnerables socialmente, con mayor índice de necesidades básicas insatisfechas, con más crecimiento y densidad poblacional de la ciudad de Santa Fe. Podría inferirse que la presencia de barreras culturales, geográficas, económicas, sociales y comunicacionales, etc. estaría impidiendo o limitando el acceso de las mujeres a los medios de tamizaje. De este modo, se estaría debilitando la participación de las mismas en el ejercicio de su derecho a la salud. En este cuatrienio, en el Centro de Salud San Agustín, se tomó un total de 318 PAP. Sólo se informó desde el Laboratorio de Papanicolaou: 7 ASCUS $^{2}$ y 2 L- SIL. ${ }^{3}$ Esto da cuenta de que el tamizaje —además de ser bajo en número- se está realizando en mujeres muy jóvenes: el 35 \% de los PAP corresponde a menores de 25 años. Si bien el $65 \%$ fue realizado en mujeres entre 25 a 64 años, de este porcentaje, el $80 \%$ fue realizado en mujeres de 25 a 30 años. Esta franja etaria sigue siendo de mujeres jóvenes. Justamente en el resto —mayores de 35 años— es donde está comprobado, por evidencia científica y por la historia natural de la enfermedad, que se encontrarían o detectarían tempranamente las lesiones preneoplásicas ${ }^{4}$ o el cáncer. Por tal motivo este proyecto se orienta hacia aquellas mujeres de 35 a 64 años pertenecientes a los barrios San Agustín. ${ }^{5}$ Sin embargo, cabe aclarar que este proyecto contempla trabajar preventivamente con las otras franjas etarias, aunque no estén en situación de riesgo.

A partir de la nominalización de las mujeres realizada en el presente

actualizados. Si bien se puede disponer de datos censales del año 2001 por Vecinal, no sería prudente realizar una proyección, ya que se trata de una de las poblaciones con mayor índice de natalidad además de haber sufrido, en los últimos años, un exponencial crecimiento poblacional, por migraciones internas, asentamientos, etc. Se buscó información a partir del censo 2011, por Vecinal, pero todavía no está procesado, según datos del IPEC. 
año en el Centro de Salud San Agustín, se puede decir que esta institución consta de un total 1404 mujeres, de las cuales 1023 no realizaron PAP desde el año 2009 (datos que fueron cruzados con el Sistema Informático de Papanicolaou, SISPAP, que contiene información desde 2009).

Entre 30 y 64 años, 319 mujeres no han recibido el tamizaje. Más de 203 mujeres se realizaron el PAP entre los años 2009 y 2011 y deberían ser nuevamente controladas. (Ver tabla 2)

Según la población objetivo del proyecto de extensión, entre la edad de 30 a 64 años 529 no se han realizado el PAP. (Ver tabla 3 y 4)

A partir de la lectura de los datos presentados podemos decir que la población de riesgo es la que menos transita por el centro de salud para realizarse el PAP. Podría inferirse que presentan ciertas dificultades de accesibilidad al sistema de salud. Según Stolkiner,

"la accesibilidad se construye como el vínculo entre los sujetos

y los servicios. Este vínculo surge de una combinatoria entre las condiciones y discursos de los servicios y condiciones de los sujetos. Tal conjunción, se manifiesta en la modalidad particular que adquiere la utilización de los servicios"

(Stolkiner y otros, 2000:04).

En este sentido se piensa a la accesibilidad como un problema de encuentro/desencuentro entre la población y los servicios de salud, lo que implica la inclusión de las prácticas y de los discursos tanto de los profesionales de los servicios de salud como de la población que accede al servicio.

La accesibilidad, por lo tanto, no se define simplemente sólo por el acceso a una consulta a un servicio de salud. Implica tener en cuenta diferentes momentos de la accesibilidad, esto es, una accesibilidad inicial que sería el ingreso al sistema de salud y el inicio de la atención, y una accesibilidad ampliada que abarca todo el proceso de atención y el tratamiento.

Los aportes de Rossi y otros (2007), señalan diferentes dimensiones de la accesibilidad como son:

"La dimensión geográfica: se refiere a la localización del servicio de salud y a su población asignada; las distancias entre el lugar de residencia de los usuarios y el servicio de salud; el desplazamiento de poblaciones de una jurisdicción a otra para hacer uso del servicio de salud, tanto porque en su zona carecen de los mismos o porque prefieren o eligen un mejor servicio. A la cuestión de la distancia geográfica se suma un elemento de la dimensión económica: costo del transporte y tiempo de viaje" (p. 12).

En este punto, muchas mujeres expresan no realizarse los controles en el centro de salud del barrio y recurren a los hospitales públicos, porque es ahí donde reciben a sus hijos cuando son madres y por tanto deciden continuar con ese mismo médico, mientras que otras expresan recibir en los hospitales mejor atención. Un número menor de mujeres se hacen atender en clínicas privadas porque por sus parejas poseen obra social o porque consideran que la atención en el sector privado garantiza la calidad de la atención. Todos estos aspectos dan cuenta una movilidad geográfica con un costo económico que tiene que hacer la mujer desde su domicilio hasta el hospital o clínica privada en búsquedas de hacer atender su salud.

"La dimensión económica: se refiere al acceso gratuito o no, al servicio de salud" (p. 12).

Cabe señalar que la realización del PAP y el control ginecológico anual en los centros de salud pública según lo establece la Ley de Salud Sexual y Reproductiva es gratuita.

"La dimensión organizacional: abarca los aspectos burocráticos y administrativos de cada institución -modalidad de los turnos o interconsultas, tiempo de espera, etc." (p. 12).

Particularmente, en este punto se pudo realizar y actualizar la construcción de un fichero para nominalización y seguimiento de mujeres. Se llevó adelante una búsqueda activa de mujeres que asisten asiduamente al centro de salud pero que no concurren al control ginecológico, mediante la captación y oferta de un turno con el médico ginecólogo en sala de espera, en los consultorios médicos o en la oficina de enfermería. Se fue cruzando la información entre los registros del centro de salud (registros 2011-2012) y el SISPAP. Y por último se fue georreferenciando a los grupos que menos acceden al centro de salud mediante un rastrillaje ${ }^{6}$ en dichas zonas.

"La dimensión cultural: analiza la posibilidad de uso de un servicio, derivada de actitudes originadas en creencias o rasgos educacionales. Bajo esta dimensión puede indagarse sobre los hábitos, prácticas, actitudes y creencias de los actores. Como el proceso institucional se basa en una relación social entre trabajadores de la salud y población usuaria del sistema, la dimensión cultural afecta a ambos" (p. 12).

Un elemento fundante de esta dimensión es la categoría identidad de género, que como se ha mencionado en multiplicidad de estudios, alude a una dimensión que es socialmente construida e históricamente variable; que no incluye sólo el binomio mujer/ varón y sus roles de producción y reproducción social, sino más bien un inconsciente colectivo, que mueve las subjetividades de cada ser. El ser mujer traslada el movimiento inconsciente hacia los roles de cuidado: esposa, madre, hija como primordiales modos ejercer lo femenino. Esta característica y la relación histórica de subordinación del cuerpo de la mujer a la reproducción y al 
placer masculino, conlleva en sí misma una invisibilización del cuerpo femenino Las mujeres y los prestadores de salud han generado diferentes estrategias para el cuidado en el embarazo y el acompañamiento en los primeros años de vida de los hijos. Pero se repite como dato, el alejamiento progresivo de las mujeres mayores de 35 años de los servicios de salud, y la falta de acceso de aquellas que, por elección, no desean conformar el binomio madre e hijo/a o por elección sexual diferente; regresando a los mismos en la etapa de aparición de enfermedades crónicas y a veces con pocas posibilidades de tratamiento, representando éstas las oportunidades perdidas. ${ }^{7}$

En definitiva, puede verse que, por fuera de las etapas de cuidado de otros (con ello referimos a los momentos de las trayectorias femeninas en los embarazos, la lactancia y primeros años de vida de los hijos), las mujeres, sobre todo, las de los sectores más vulnerables de la sociedad; dejan de asistir a las consultas médicas y realizarse chequeos preventivos.

Otro elemento a considerar en la elaboración de este problema multidimensional, es el acceso a la información y formación respecto a las propias necesidades y derechos. La sexualidad, y con ella el cuerpo femenino, siguen siendo un tabú en muchos estratos sociales. Las mujeres no pueden (simbólica y materialmente) priorizar la atención de su salud porque estarían dedicando tiempo a lo mundano, lo secundario, lo no esencial; las mujeres como sujetos y actores reproducen, inconscientemente, la inequidad y los roles tradicionalmente adjudicados.

\section{Modalidad de abordaje:}

\section{un trabajo colectivo entre diferentes actores sociales}

La estrategia de intervención de este PEIS se pensó en dos grandes líneas: una más vinculada al trabajo con equipo de salud y otra en relación más directa con las mujeres que conforman la población objetivo.

En la primera línea se pudo capacitar tanto al equipo extensionista como al equipo del centro de salud respecto de la problemática de intervención de este PEIS. Con el primero, se construyó un marco conceptual en común a partir de las categorías: salud pública, accesibilidad, atención de población a cargo, género, cáncer de cuello uterino, estrategias de prevención, interdisciplina, trabajo en red. Con el segundo, una de las instancias de capacitación fue llevada adelante con la participación de referentes de la Secretaría de Salud de la Municipalidad de Santa Fe, quienes en el marco del Programa municipios saludables se encontraban realizando un proyecto con objetivos similares en los centros de salud Abasto, Nuevo Horizonte, Cabaña Leiva.
Esta capacitación dejó visibilizada la necesidad de un trabajo en red, interinstitucional e interdisciplinario para el abordaje de una problemática tan compleja como es el cáncer de cuello uterino. En este sentido, por un lado, la interdisciplina no trata de imponer un saber sobre otro, sino simplemente intenta que se produzca un diálogo entre diferentes saberes, entre diferentes profesionales interesados en un problema determinado, respecto del cual se pueden mantener puntos de vista distintos. Se trata pues, de un diálogo que intenta narrar, dar sentido, recoger, reunir lo que aparece como común entre los profesionales que integran un equipo. En esta perspectiva, se fue construyendo un espacio de trabajo conjunto entre personal del centro de salud e integrantes del equipo extensionista, para la planificación y seguimiento de las acciones a seguir en el marco del PEIS, y poner en marcha nuevas estrategias de intervención para la mayor captación de mujeres: nominalización, recupero de oportunidades perdidas, mayor oferta de servicio para la toma de PAP. Acciones que tienen la finalidad de ser sustentables en el tiempo y dar respuestas necesarias a todas las mujeres tanto en la toma de muestras como en el seguimiento de las citologías positivas.

Por otro lado, el trabajo en red alude a los vínculos que se establecen entre personas, grupos y/o instituciones que, con sus propias características y particularidades, reconocen la necesidad de crear lazos y aunar esfuerzos para el cumplimiento de objetivos comunes. En este sentido, según Rovere (2006) redes es un concepto vincular, "es el lenguaje de los vínculos". Desde esta concepción, para la capacitación se convocó al personal de otros centros de salud y a organizaciones sociales del barrio San Agustín. Se tomó contacto con los Centros de Extensión Comunitaria Distrito Noroeste de la UNL, se participó de la reunión de la red del barrio y se realizó un relevamiento de organizaciones, redes y fuerzas vivas de la zona de influencia del centro de salud en las cuales participen mujeres. Para el trabajo más directo, en el marco del día de la salud de la mujer, se organizó en forma conjunta con el equipo del centro de salud talleres creativos y lúdicos con las mujeres del barrio (una mateada de salud para la mujer). Actividad que implicó planificación, difusión, trabajo en equipo y red para la convocatoria de mujeres. Cabe aclarar que, este espacio y los próximos que tiene planificado el proyecto, tienen como fin promover, por un lado, un vínculo más estrecho entre centro de salud y la comunidad y, por otro lado, recuperar y potenciar capacidades en las mujeres para que, autónomamente, puedan resolver sus problemáticas y, por último, promover la participación y el cuidado de la salud desde una perspectiva de derecho y de equidad de género. mujeres del rango etario seleccionado concurren asiduamente al centro de salud a diferentes servicios, consultas o por cualquier prestación, pero nadie

las visualiza para la prevención de cáncer de cuello uterino. 


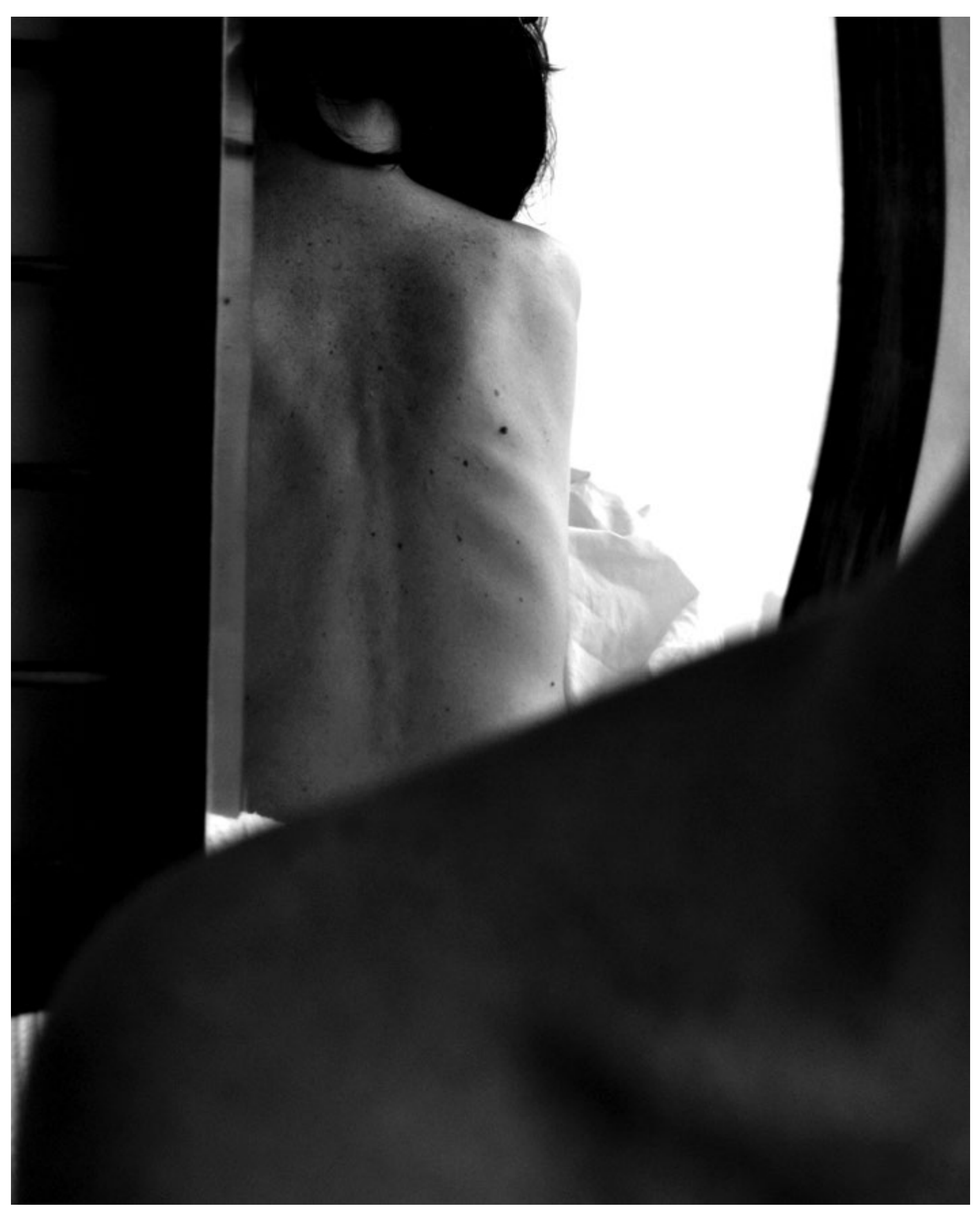




\section{Bibliografía}

Arrossi, S y Paolino, M (2008). Proyecto para el mejoramiento del Programa Nacional de Prevención del Cáncer de Cuello Uterino en Argentina. Buenos Aires, Editorial OPS. Arrossi, S. y otros (2007). The socio-economic impact of cervical cancer on patients and their families in Argentina, and its influence on radiotherapy compliance. Results from a cross- sectional study. Editorial Gynecologic Oncology.

Ferlay, J y otros (2004). Cancer Incidence, Mortality and Prevalence Worldwide Version 2.0.IARC Cancer Base N ${ }^{\circ}$ 5. Lyon, IARC.

Ministerio de Salud de la Provincia de Santa Fe (2012). Informe sobre datos provenientes del Laboratorio de Papanicolaou Dr. Daniel Rocco del Ministerio de Salud de la Provincia; en el período 2009-2012 a partir del SISPAP (Sistema Informático de Papanicolaou de la Provincia).

Ministerio de Salud de la Provincia de Santa Fe (2014). Informe sobre nominalización de mujeres Centro de Salud San Agustín realizado por el Laboratorio de Paps de la Provincia.

Rovere, M. (2006). Redes en Salud; los grupos, las instituciones, la comunidad.
Córdoba, Editorial El Agora

Proyecto de Extensión de Interés Social (2014). Informe de avance del proyecto "Prevención de Cáncer de Cuello Uterino: estrechando lazos entre el equipo de salud y las mujeres. Experiencia de trabajo en el Centro de Salud San Agustín". Rossi, D.; Pawlowicz, M.P.; Zunino Singh, D. (2007). Accesibilidad de los usuarios de drogas a los servicios públicos de salud en las ciudades de Buenos Aires y Rosario: la perspectiva de los trabajadores de la salud. Buenos Aires, Intercambios Asociación civil.

Stolkiner, A. (1999a). "Interdisciplina II: Lo transdisciplinario como momento o como producto" en El CAMPO Psi: Facultad de Psicología. Buenos Aires. Stolkiner, A. (1999b). "La Interdisciplina: entre la epistemología y las prácticas" en El CAMPO Psi: Facultad de Psicología. Buenos Aires.

Stolkiner, A. y otros (2000). "Reforma del Sector Salud y utilización de servicios de salud en familias NBI: estudio de caso" del libro La Salud en Crisis - Un análisis desde la perspectiva de las Ciencias Sociales, Buenos Aires, Ed. Dunken.

\section{Tabla 1}

\section{Producción PAP Centro de salud San Agustín}

\begin{tabular}{|c|c|c|c|c|c|}
\hline Año & > 25 años & 25-64 años & $<64$ años & Pap 1 vez & Totales \\
\hline 2009 & 15 & 45 & 0 & 0 & 60 \\
\hline 2010 & 20 & 76 & 1 & 0 & 97 \\
\hline 2011 & 17 & 79 & 1 & 1 & 97 \\
\hline 2012 & 22 & 72 & 0 & 5 & 94 \\
\hline
\end{tabular}

Fuente: SISPAP (Sistema Informático de Papanicolaou de la Provincia).
Tabla 2

\begin{tabular}{|c|c|}
\hline Año & $\begin{array}{l}\mathbf{N}^{\circ} \text { de mujeres } \\
\text { con paps }\end{array}$ \\
\hline 2007 & 1 \\
\hline 2009 & 20 \\
\hline 2010 & 38 \\
\hline 2011 & 62 \\
\hline 2012 & 85 \\
\hline 2013 & 106 \\
\hline 2014 & 66 \\
\hline
\end{tabular}

Tabla 3

\begin{tabular}{|c|c|}
\hline Grupo de edad & $\begin{array}{l}\mathrm{N}^{\circ} \text { de mujeres } \\
\text { con paps }\end{array}$ \\
\hline 13-15 años & 129 \\
\hline 16-20 años & 341 \\
\hline 21-25 años & 193 \\
\hline 26-29 años & 97 \\
\hline 30-64 años & 529 \\
\hline 65 y más & 81 \\
\hline Sin datos & 34 \\
\hline
\end{tabular}

\section{Tabla 4}

Laboratorio de Procesamiento

de Papanicolau Dr. Daniel Rocco

Análisis de Resultados por edades
Período de recepción de muestra: Desde: 01-01-2014 Hasta: 24-07-2014 Centro de toma de muestra: CS San Agustín - La Capital Patólogos: Todos

\begin{tabular}{|c|c|c|c|c|c|c|c|c|c|c|c|c|c|c|c|c|}
\hline Diagnósticos por Calidad de Muestra & $>15$ & $15 / 19$ & $20 / 24$ & $25 / 29$ & $30 / 34$ & $35 / 39$ & $40 / 44$ & $45 / 49$ & $50 / 54$ & $55 / 59$ & $60 / 64$ & $65 / 69$ & $>70$ & S/E & Total & \\
\hline Insatisfactorios & 0 & 0 & 0 & 1 & 0 & 1 & 1 & 0 & 0 & 0 & 1 & 0 & 0 & 0 & 6 & $3,35 \%$ \\
\hline Satisfactorios & 0 & 10 & 42 & 31 & 19 & 29 & 23 & 12 & 5 & 3 & 4 & 0 & 0 & 0 & 173 & $96,65 \%$ \\
\hline & & & & & & & & & & & Total d & citolo & as ev & uadas & 179 & $100 \%$ \\
\hline
\end{tabular}

Detalle de los Diagnósticos Satisfactorios seleccionados

\begin{tabular}{|c|c|c|c|c|c|c|c|c|c|c|c|c|c|c|c|c|}
\hline Diagnósticos por grupos de Satisfactorios & $>15$ & $15 / 19$ & $20 / 24$ & $25 / 29$ & $30 / 34$ & $35 / 39$ & $40 / 44$ & $45 / 49$ & $50 / 54$ & $55 / 59$ & $60 / 64$ & $65 / 69$ & $>70$ & S/E & Total & \\
\hline Ascus & 0 & 0 & 0 & 1 & 0 & 1 & 1 & 0 & 0 & 0 & 1 & 0 & 0 & 0 & 6 & $1,73 \%$ \\
\hline Ausencia de células neoplásticas & 0 & 10 & 41 & 31 & 19 & 23 & 22 & 10 & 5 & 3 & 4 & 0 & 0 & 0 & 168 & $97,11 \%$ \\
\hline \multirow[t]{2}{*}{ L-SIL } & 0 & 0 & 1 & 0 & 0 & 0 & 0 & 1 & 0 & 0 & 0 & 0 & 0 & 0 & 2 & $1,16 \%$ \\
\hline & & & & & & & & & & \multicolumn{5}{|c|}{ Total de citologías satisfactorias } & 173 & $100 \%$ \\
\hline
\end{tabular}

${ }^{*}$ ) S/E hace referencia a los casos "Sin Edad" debido al desconocimiento de la fecha de nacimiento del paciente. 